\title{
Improved Models for the Spread of Ebola
}

\author{
Liye Xie
}

North China Electric Power University, Baoding 071000, China

Keywords: Epidemic model, improved SEIR model.

\begin{abstract}
The Ebola epidemic is more and more severe. To optimize the eradication of EVD, we take Liberia, Guinea and Sierra Leone as the object of research, first we build suspected- exposed-I nfectious-dead (SEID) model based on the popular SEIR model, since there are no effective medicine till now and the mortality rate of EVD is pretty high. It turns out that the curve of SEID agrees with actual data very well. Then we establish a suspected-exposed-infectious-quit (SEIQ) model by modifying the previous SEID model, which means that infected people quit the class either recovered or dead. Then we address the SEIQ problem and conclude that the growth rate of infectious people decreased obviously when medicine plays a part.
\end{abstract}

\section{Introduction}

The Ebola outbreak in West Africa was first reported in March 2014, and has rapidly become the deadliest occurrence of the disease since its discovery in 1976.In fact, the current epidemic sweeping across the region has now killed more than all other known Ebola outbreaks combined.Up to 2 February, 9,019 people had been reported as having died from the disease in six countries; Liberia, Guinea, Sierra Leone, Nigeria, the US and Mali.1So controlling the Ebola epidemic becomes a burning issue which brooks no delay.

\section{Modeling the spread of Ebola}

\subsection{Symbols and Definitions}

Table 1 Symbols

\begin{tabular}{cc}
\hline Symbol & Definition \\
\hline $\mathrm{N}(t)$ & The total popularity of Liberia, Guinea, Sierra Leone \\
$E(t)$ & The fraction of susceptible people at time $t$ \\
$I(t)$ & The fraction of exposed people at time $t$ \\
$D(t)$ & The fraction of infective people at time $t$ \\
$\lambda$ & The fraction of died people at time $t$ \\
$q$ & The average number of contacts with an infective people per day \\
$\beta$ & The probability that an infectious people can be found and isolated \\
$\varepsilon$ & The morbidity of people in the latent period in a day \\
$\mathrm{g}$ & The death rate per day \\
$R(t)$ & The fraction of recovered people at time $t$ \\
$f$ & The death rate \\
$C$ & Healing period of the medicine \\
$M(t)$ & Manufacturing speed \\
$\alpha$ & The transportation efficiency, range from 0 to1 \\
$A$ & Adequacy of the medicine \\
$t_{1}$ & The turning point of manufacturing \\
\hline
\end{tabular}




\subsection{The SEID model}

The first problem we need to solve is an infectious diseases model. The infectious diseases model is a tool which has been used to study the mechanisms by which diseases spread, to predict the future course of an outbreak and to evaluate strategies to control an epidemic (Daley \&Gani, 2005).5 As for Ebola,it has characters as below:

- it influences a large number of popularity, and

- it has what's called latent period, during which the individual is said to be infected but not infectious. So the SEIR (susceptible-exposed-infectious-recovered) model is most close to the Ebola disease, but we assume that infective people of Ebola will all die, that is to say, recovery will not happen in this situation, so we modify the SEIR to SEID (susceptible-exposed-infectious-dead) model. In this model, individuals in the population are assigned to different subgroups,

each representing a specific stage of the epidemic. Moreover, the transition rates from one class to another are mathematically expressed as derivatives, hence the model is formulated using differential equations. In this model the host population $(\mathrm{N})$ is broken into four compartments: susceptible, exposed, infectious, and died, with the numbers of individuals in a compartment, or their densities denoted respectively by $\mathrm{S}(\mathrm{t}), \mathrm{E}(\mathrm{t}), \mathrm{I}(\mathrm{t}), \mathrm{D}(\mathrm{t})$, that is $1=\mathrm{S}(\mathrm{t})+\mathrm{E}(\mathrm{t})+\mathrm{I}(\mathrm{t})+\mathrm{D}(\mathrm{t})$.

As is shown in Figure 1, susceptible individuals $\mathrm{S}$ become infected by corpses and infectious individuals who haven't been isolated. They then move through an latent period (E) at rate $\varepsilon$ before they become infectious individuals I. Infectious individuals die at rate $g$.

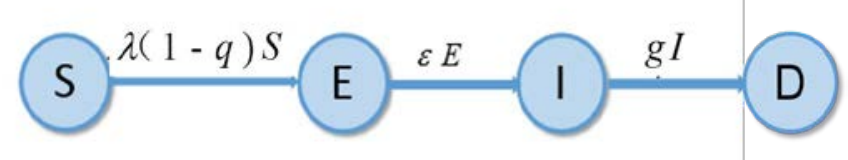

Figure 1. Schematic illustration of the EVD spread model

Thus EVD transmission can be described by the following set of differential equations:

$$
\begin{aligned}
& \left\{\begin{array}{l}
\frac{d S}{d t}=-\lambda I(1-q) S-\beta D S \\
\frac{d E}{d t}=\lambda I(1-q) S+\beta D S-\varepsilon E \\
\frac{d I}{d t}=\varepsilon E-g I \\
\frac{d D}{d t}=g I
\end{array} \quad(t<200)\right. \\
& \left\{\begin{array}{l}
\frac{d S}{d t}=-\lambda I(1-q) S \\
\frac{d E}{d t}=\lambda I(1-q) S-\varepsilon E \\
\frac{d I}{d t}=\varepsilon E-g I \\
\frac{d D}{d t}=g I
\end{array} \quad(t \geq 200)\right.
\end{aligned}
$$

After infection, susceptible individuals $S$ enter the exposed class $E$ before they become infectious individuals I and die (D). Consider that people may have no idea that corpses can be a source of infection and didn't take preventive measures, we added this influence in our equations. After the implementation of control measures, i.e.when $t \geq 200$, we ignore this factor which may cause infection.

\subsection{The SEIQ model}

Once if there are effective medicine or vaccine for Ebola, the situation will be enormously improved in West Africa. A proportion of patients will be recovered rather than only waiting for 
death. Thus we conduct a new model called SEIQ (susceptible-exposed-infectious-quit) based on our SEID model. The construction of SEIQ model is shown in Figure 2.

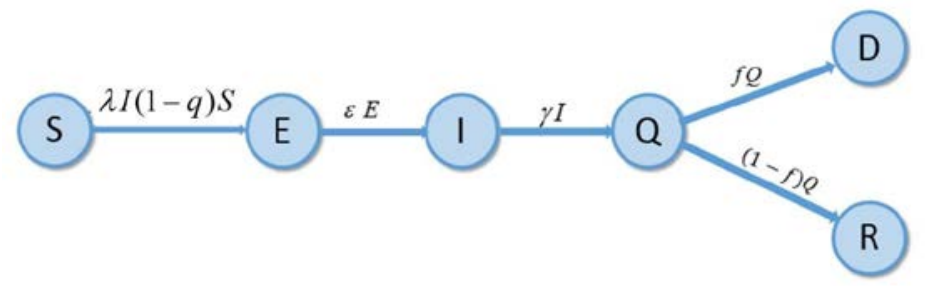

Figure 2 the construction of SEIQ model

The difference between SEIQ and SEID:Since there are effective medicines, a part of people will quit the infectious state every day, either dead or recovered, and the fatality rate is given by $f$.

The equations of SEIQ can be expressed as follows:

$$
\begin{aligned}
& \left\{\begin{array}{l}
\frac{d S}{d t}=-\lambda I(1-q) S \\
\frac{d E}{d t}=\lambda I(1-q) S-\varepsilon E \\
\frac{d I}{d t}=\varepsilon E-\left(g^{\prime}+r^{\prime}\right) I \\
\frac{d Q}{d t}=\left(g^{\prime}+r^{\prime}\right) I
\end{array}\right. \\
& \left\{\begin{array}{l}
\frac{d S}{d t}=-\lambda I(1-q) S \\
\frac{d E}{d t}=\lambda I(1-q) S-\varepsilon E \\
\frac{d I}{d t}=\varepsilon E-\left(g^{\prime \prime}+r^{\prime \prime}\right) I \\
\frac{d Q}{d t}=\left(g^{\prime \prime}+r^{\prime \prime}\right) I
\end{array}\right.
\end{aligned}
$$

Where $t_{1}$ is the turning point we need to figure now.

In stage 1 , we know that $g^{\prime}=0.9, r=P A+g^{\prime}$,plugging these two equations, we can draw the trace of $I(t)$ and $M(t)$ in a same image as in figure 3.

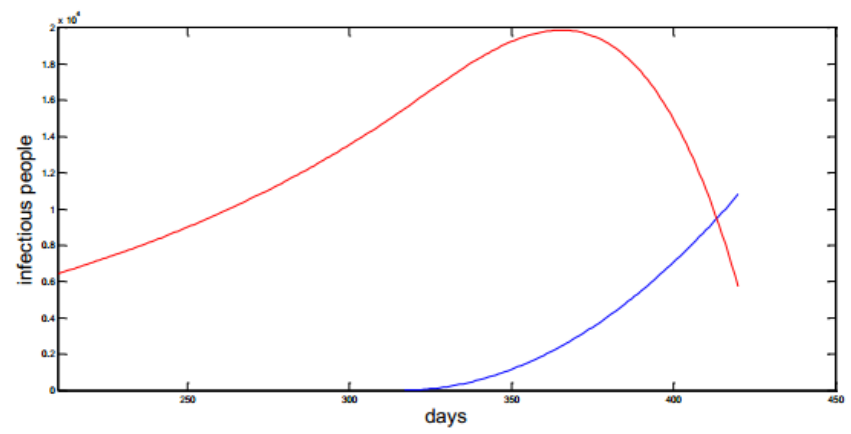

Figure 3 finding the turning point

As we can see, there is a crossover of the red line and blue line, which is the turning point we need. The time of that turning point is 414 days after the outbreak, on the base of this we can do the next step. 


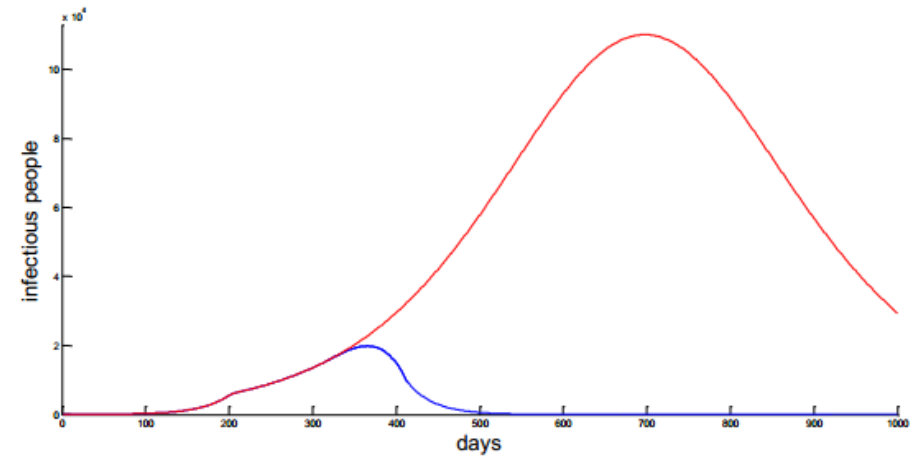

Figure 4 the effect of medicine

\section{Conclusion}

- If the use of drugs to prevent and treat the epidemic will spread rapidly, the number of patients will be the first 383 days to reach the peak with more than 11 million.

- if taking medicine, the epidemic spread speed will slow, the number of patients is only close to the peak at 2 million and the epidemic and is expected to be eliminated at about the 278th day.

- Therefore, in order to eradicate the Ebola virus, the effects of medicine is essential.

\section{References}

[1]. Information on : http://www.bbc.co.uk/news/world-africa-26835233

[2]. Information on: http://en.wikipedia.org/wiki/Liberia

[3]. Information on:http://www.washingtontimes.com/news/2014/aug/8/who-ebola-outbreak-is-a-pu blic-health-emergency/?page=allInformationn :http://www.cdc.gov/mmwr/preview/mmwrhtml/ mm6344a6.htm

[4]. Information on :http://en.wikipedia.org/wiki/Epidemic_model

[5]. Information on :http://en.wikipedia.org/wiki/Kruskal\%27s_algorithm 